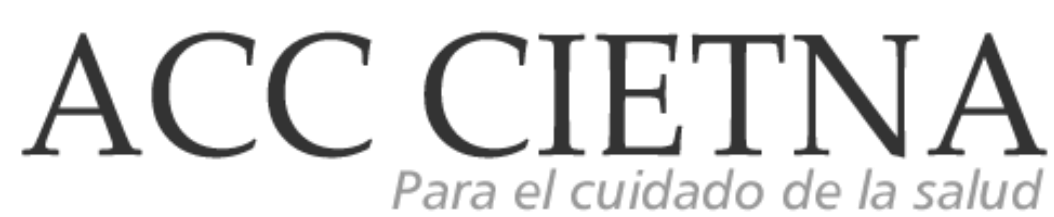

https:// doi.org/10.35383/cietna.v6i2.261

\title{
El uso de un lactario público, desde las experiencias de las madres
} usuarias

\author{
Kyara Yahyrza, Guadalupe ${ }^{1}$, Mogollón Torres, Flor de María ${ }^{2}$
}

INFORMACIÓN DEL ARTÍCULO RESUMEN

\section{Historia del artículo:}

Recibido el 11 de septiembre de 2019

Aceptado el 15 de noviembre de 2019

Palabras clave:

Bancos de Leche

Experiencias de Vida

Leche materna
Investigación cualitativa, descriptiva, cuyo objetivo fue discutir la utilidad de un lactario público, desde las experiencias de las madres usuarias. La muestra fue no probabilística por conveniencia y el tamaño se determinó por saturación y redundancia; lo constituyó 15 madres, que hicieron uso del lactario del centro comercial Real Plaza, de Chiclayo; que tenían niños de 0 a 2 años, independientemente del tipo de lactancia que practicaron y que aceptaron voluntariamente participar del estudio. Los datos fueron recolectados mediante la técnica de la entrevista semiestructurada y el instrumento fue la guía de entrevista, elaborada por las investigadoras y validada por juicio de expertos y prueba piloto una vez aprobado el proyecto por el Comité de Ética en Investigación de la Facultad de Medicina de la USAT. Los datos se trataron mediante el análisis de contenido, y en toda la investigación se tuvo en cuenta criterios éticos propuestos por Sgreccia y de rigor científico por Noreña. Obteniendo como resultados dos categorías: Uso adecuado del lactario vs uso inadecuado del lactario. Arribando a la consideración general: que existe un uso adecuado vs inadecuado del lactario público y limitantes para el uso, como: la infraestructura, el desconocimiento del uso correcto por falta de difusión y monitoreo frente a la cual las investigadoras recomiendan difundir la normativa vigente en cuanto a la implementación y funcionamiento del lactario.

\footnotetext{
'Licenciada en Enfermería en Centro de Salud Mochumí, Lambayeque, Perú. Email: yahyrzacp@gmail.com

2Magister en Enfermería. Docente de la Escuela de Enfermería de la Universidad Católica Santo Toribio de Mogrovejo. Enfermera especialista en Enfermería Materno Infantil con Mención en Neonatología, del Centro de Salud Toribia Castro Chirinos, Lambayeque, Perú. Email: fmogollon@usat.edu.pe. ORCID: https://orcid.org/0000-0002-2605-546X
} 
The use of a public lactarium, from the experiences of user mothers

ABSTRACT

Keywords:

Milk Banks

Life experiences,

Breast milk
Qualitative, descriptive research, whose objective was to discuss the usefulness of a public lactarium, from the experiences of user mothers. The sample was not probabilistic for convenience and the size was determined by saturation and redundancy; it was constituted by 15 mothers, who made use of the lactarium of the Real Plaza shopping center, in Chiclayo; that they had children from 0 to 2 years old, regardless of the type of breastfeeding they practiced and who voluntarily agreed to participate in the study. The data were collected using the semi-structured interview technique and the instrument was the interview guide, prepared by the researchers and validated by expert judgment and pilot test once the project was approved by the Research Ethics Committee of the Faculty of Medicine of the USAT. The data were treated through content analysis, and throughout the investigation, ethical criteria proposed by Sgreccia and scientific rigor by Noreña were taken into account. Obtaining two categories as results: Proper use of the lactarium vs. improper use of the lactarium. Coming to the general consideration: that there is an adequate vs inadequate use of the public lactarium and limitations for the use, such as: infrastructure, lack of knowledge of the correct use due to lack of dissemination and monitoring against which the researchers recommend disseminating current regulations in regarding the implementation and operation of the lactarium.

\section{Introducción}

Desde la existencia de la humanidad, la leche materna se ha constituido como la principal fuente de alimento para el lactante. De esta forma, debemos decir que la leche humana es el alimento idóneo para las necesidades de crecimiento y desarrollo de la especie humana. Tal es así que las organizaciones internacionales que se ocupan durante años de la alimentación del recién nacido y el lactante, preconizan que la lactancia materna es el mejor alimento para el recién nacido y el lactante hasta el primer año de vida $^{1,2}$.

Asumir el mantenimiento de la lactancia natural por parte de madres trabajadoras que se reincorporan a su función fuera del hogar, durante el periodo recomendado, no solo es decisión y disposición de la madre, sino que requiere ayuda y una buena planificación tanto laboral como familiar. De esta manera, la madre debe prepararse desde el embarazo, conociendo las medidas que tiene su empleador para el personal que amamanta, como: la presencia de un lugar apropiado donde podría amamantar a su bebé con las debidas medidas de bioseguridad y la privacidad necesarias, los horarios disponibles, los equipos y materiales que se requieren para la práctica de la lactancia materna, etc ${ }^{1,3}$.

Debemos indicar que, la ocupación laboral de la mujer y su participación en el proceso económico es necesaria para ella y la sociedad, y la reincorporación a la vida laboral no justifica el abandono de la lactancia materna. Por ello, es 
indispensable un cambio en nuestra cultura, que priorice el factor humano, es decir, se haga énfasis en la humanización de los ambientes laborales y en el desarrollo de una cultura de la lactancia materna exclusiva y prolongada 3 .

En tal sentido, existe el compromiso del Estado Peruano de asegurar que todos los sectores de la sociedad, y en particular las madres, conozcan las grandes ventajas del amamantamiento. Tal compromiso llevó al gobierno, a promulgar mediante Ley $\mathrm{N}^{\circ} 29896$ y Decreto Supremo $\mathrm{N}^{\circ}$ 009-2006-MIMDES, la implementación de lactarios en las instituciones del sector público y privado, a fin de promover la lactancia materna en los centros de trabajo, en el marco de las políticas de conciliación entre la vida familiar y las actividades laborales ${ }^{4}$.

Es así, que ciertas instituciones a nivel nacional y local vienen implementando dichos lactarios, algunos de estos cumpliendo con lo estipulado en la normativa; en tanto que otros, lo realizan según sus posibilidades y criterios. Sin embargo, existe una gran cantidad de instituciones, que, aun presentando las condiciones y las exigencias del caso, no cuentan con un lactario; a ello se suma, que la mayoría de sus trabajadoras desconocen los derechos que les asiste en cuanto a la práctica del amamantamiento, frente a lo cual optan por la interrupción de la lactancia materna exclusiva y la introducción de sucedáneos de la lecha materna, cuando se reinsertan a su centro laboral.

Hoy se cuenta con 1502 lactarios implementados en instituciones públicas y privadas, cifra que representa un gran avance en la institucionalización de políticas públicas sobre igualdad de género, promoción de la lactancia materna, conciliación de la vida familiar con el trabajo y las vinculadas al derecho de un empleo digno 5 .

Ante la realidad descrita en los párrafos anteriores, surgió en las investigadoras, la necesidad de indagar y sistematizar la información, acerca de las diferentes experiencias que tienen las madres sobre el uso del lactario, en uno de los establecimientos de mayor concurrencia en nuestra localidad, como lo es el centro comercial Real Plaza, al que acuden gran cantidad de madres que hacen uso de dicho espacio, por diferentes motivos, y no necesariamente por el de amamantamiento.

Siendo así que, en una primera inmersión realizada por una de las investigadoras al escenario, y al preguntarles sobre si creen que dicho lactario cumple con las normativas vigentes, contestaron desconocer las normativas. De este modo, la población al no conocer las normativas relacionadas a la instauración y funcionamiento de los lactarios, no exige la implementación de nuevos lactarios institucionales y su correcto funcionamiento; por lo que, las investigadoras creyeron conveniente, indagar sobre el uso que le vienen dando al único lactario público de la ciudad de Chiclayo, desde la experiencia de las mismas personas que acuden diariamente a dicho ambiente, planteándose la siguiente interrogante de investigación: ¿Cómo es el uso del lactario público, desde la experiencias de las madres usuarias? Planteándose como objetivo: discutir la utilidad de un lactario público, desde las experiencias de las madres usuarias.

El presente estudio se justificó porque a nivel nacional la implementación de los lactarios, es una exigencia respaldada por una normativa vigente emanada del Ministerio de Salud (MINSA), que rige tanto al sector público como privado; pues se constituye en una estrategia para promocionar la práctica de la lactancia materna, frente a las exigencias de la gran cantidad de madres que desarrollan funciones fuera del hogar, como trabajadoras; por tanto la temática es de gran relevancia social, puesto que la existencia e importancia del funcionamiento del lactario en los escenarios públicos y privados, es una forma de ejecutar las políticas de promoción y protección de la lactancia materna, además de velar por la economía de las madres, en la que estas no se vean obligadas a recurrir al pago de servicios extras, concretizando así una de las metas del Estado ${ }^{6}$.

Por otro lado, el presente trabajo de investigación, también servirá como antecedente para otras investigaciones que se relacionen con el 
objeto de estudio, por ser un tema inédito y coyuntural a nivel local y nacional.

\section{Metodología}

La investigación fue cualitativa- descriptiva ${ }^{7,8}$, con abordaje metodológico estudio de caso $^{9}$, porque se buscó comprender y profundizar en el fenómeno, en lo que las participantes que acudieron al lactario manifestaron, pensaron, sintieron y vivieron, así como el proceso y significado de sus relaciones interpersonales con las investigadoras y el entorno, tratando de comprender las experiencias, percepciones y puntos de vista, que estas tienen frente al uso del lactario, independientemente de los prejuicios de las investigadoras.

Los sujetos de investigación ${ }^{10}$, fueron captados en el lactario del Centro Comercial Real Plaza Chiclayo y se caracterizaron por ser: madres usuarias, cuyas edades oscilaron entre los 20 a 39 años de edad; con nivel de instrucción en su mayoría superior completa desempeñándose como profesoras, abogadas, licenciadas en comercio y contadoras; otras con un grado superior incompleto (estudiantes) y una minoría con secundaria completa, siendo la ocupación principal, amas de casa; en relación al lugar de procedencia el $100 \%$ eran del distrito de Chiclayo y el $100 \%$ profesan la religión católica; las edades de los hijos lactantes estuvieron entre el rango de 3 a 16 meses, el número de hijos que tenían variaba entre uno a dos niños. La muestra fue no probabilística por conveniencia, y el tamaño se determinó por criterios de saturación y redundancia ${ }^{10}$.

Los datos se recolectaron mediante la técnica de la entrevista semiestructurada 10,11 , cuyo instrumento fue una guía de entrevista, elaborada por las investigadoras y validada mediante juicio de expertos, a partir del cual se hizo la adecuación cultural necesaria.
Los sujetos se captaron en el mismo lactario, previo permiso de la dirección del Centro Comercial, a quienes se les solicitó la asignación de un ambiente privado para el desarrollo de la entrevista; el cual fue denegado, por lo que se decidió realizar las entrevistas en el domicilio de los sujetos. Una vez establecidos los permisos respectivos, se procedió a contactar en el mismo escenario a las madres usuarias que cumplían con los criterios de inclusión, estableciéndose con ellas la fecha, hora y lugar para las entrevistas, previa explicación a detalle de la investigación y la firma del consentimiento informado. La entrevista estuvo dirigida por la investigadora principal y el tiempo para cada una, fue en promedio 30 minutos; a su vez los relatos se registraron utilizando un grabador de voz, a fin de captar la totalidad de datos manifestados ${ }^{10}$ Se regresó al escenario, las veces necesarias hasta completar el tamaño de la muestra, por saturación y redundancia.

Los datos fueron procesados mediante el análisis de contenido, de manera manual. Este análisis se llevó a cabo según las etapas, propuestas por Burns ${ }^{12}$, el Pre análisis en esta se seleccionaron los datos pertinentes con el objeto de estudio, permitiéndole a las investigadoras tener una idea general de los resultados y establecer una posible esquematización de los mismos. La siguiente fase, denominada codificación, consistió en codificar la información, fragmentándola para facilitar la categorización. Finalmente, la fase de categorización, en donde se agruparon las unidades que son similares en cuanto a su significado, obteniéndose categorías y subcategorías teóricas.

Durante el desarrollo de la investigación también se pusieron en práctica los principios de la Bioética personalista sustentados por Sgreccia ${ }^{13}$, los sujetos de investigación, en todo momento fueron consideradas como personas en su totalidad de valor, respetando sus experiencias, opiniones, 
percepciones y creencias culturales que enriquecieron los resultados de la investigación, sin violentar todo tipo de derecho inherente a su persona. Se aseguró que la identidad de los sujetos este protegida en todo momento y para ello a cada quien se le asignó un código: MU1, MU2...MU15 y así sucesivamente hasta completar la muestra.

Así mismo, las participantes conocieron los procedimientos que se utilizaron para obtener la información completa, de tal forma que les permitió tomar decisiones, entre ellas firmar el consentimiento informado, garantizando así su participación voluntaria y responsable.

En esta investigación se pretendió velar, para que los datos estén completos y sean de calidad. Para ello, se tuvo en cuenta los criterios según Noreña ${ }^{14}$, credibilidad, confirmabilidad o auditabilidad y transferibilidad o aplicabilidad.

\section{Resultados, análisis y discusión}

I. El uso adecuado vs el uso inadecuado del lactario

El término uso, hace referencia a hacer servir una cosa para algo, ejecutar o practicar algo habitualmente ${ }^{15}$. Además, para diferenciar lo adecuado de lo inadecuado, establecemos los siguientes significados, sabiendo que lo primero hace referencia a lo ideal o idóneo para alguien o algo; en cambio lo inadecuado, es lo no apropiado a las condiciones $u$ objetivos de un fin ${ }^{16}$.

Por otro lado, se considera lactario, al "área delimitada cuyo único objetivo es la extracción, recepción de la leche natural y su conservación bajo parámetros adecuados, para que las madres impedidas de lactar a sus hijos, puedan proporcionar este alimento natural a los niños lactantes"6.

Es importante resaltar que la lactancia materna hoy en día se ha convertido en uno de los temas más importantes en la población, ya que favorece no solo al lactante, y a la madre; sino también, se ve beneficiada la sociedad como tal 17 .
En tal sentido, el uso que le vienen dando las madres usuarias al lactario, es diversa, lo que dio lugar a esta primera categoría, en la que se refleja una fuerte divergencia, ya que un grupo le da un "uso adecuado", como lo reportaron las madres en sus discursos:

“...Al lactario ingresé para poderle dar de lactar a mi bebé, ya que es un tanto incomodo estar en las mesitas, el tener que sacar la mama y bueno a veces que estén mirándome, entonces por eso vengo acá a este espacio para poderle dar de lactar a mi bebito..." (MU4).

“...yo ingresé al lactario, porque me fui de compras y la verdad mi niño necesitaba lactar y tuve que ocupar el lactario..., es necesario y uno no puede estar a disposición del público, es algo privado..." (MU9).

“...yo ingresé a este ambiente por darle de lactar a mi bebé, me parece un ambiente adecuado y más privado para la lactancia materna..." (MU10).

Así pues, los discursos reflejan que estas madres usuarias reconocen y dan el uso correcto al lactario, como el espacio donde pueden proporcionar leche materna a sus hijos en periodo de lactancia. Reconociendo de manera unánime que la lactancia materna es el método más seguro de alimentar a un lactante.

La Organización de las Naciones Unidas para la Agricultura y la Alimentación, y la Organización Mundial de la Salud (FAO/ OMS), recomiendan la lactancia materna exclusiva durante los seis primeros meses de vida, la cual debe continuar con adición de alimentos complementarios desde los seis meses hasta los dos años ${ }^{18}$, independientemente de que la madre trabaje o estudie, y en este sentido, los lactarios, facilitan dicha práctica.

Por otro lado, existe un grupo de madres que le dan un uso inadecuado al lactario. 
Lo que se evidencia en los siguientes discursos:

“.. nos ayuda a tener un lugar donde poder cambiar el pañal o a sentarnos un ratito con los bebes, porque ellos a veces se aburren y los podemos sentar para que jueguen..." (MU6).

"...fue de una manera muy urgente que utilicé el lactario por una necesidad de mi pequeño hijo, utilicé el servicio para el cambio de pañales, mi niño tiene un 1 año y 3 meses, ingresé solo para el cambio de pañal, exclusivamente para eso..." (MU8). “...yo ingresé al lactario porque tengo 2 gemelos, justamente a cambiarlos de pañal, porque mejor dicho se habian ocupado y necesitaba un espacio donde los podría cambiar, entonces me dirigía a los baños y mire a la mano derecha, allí estaba el lactario, entonces..." (MU5).

Discursos que dejan entrever claramente, que existe un desconocimiento total del propósito de este servicio, toda vez que lo toman como un ambiente para descanso, juego y para el cambio de pañal de los niños, lo cual refleja un conjunto de prácticas inadecuadas no estipuladas en la normativa.

Según Núñez ${ }^{19}$, existe una gran desinformación en lactancia materna, situación agravada por la existencia de múltiples factores que conspiran en general contra la lactancia natural, tales como la presión social, la influencia detractora de mitos o leyendas urbanas, la publicidad de las empresas que elaboran sucedáneos de la leche materna y la falta de apoyo oportuno a las madres.

En tanto, otro grupo de madres le dan un "uso adecuado e inadecuado a la vez", puesto que ingresan para dar de lactar y al mismo tiempo para realizar otras actividades, lo cual se demuestra en lo versado:

“...Yo ingresé al lactario porque mi niño estaba llorando, y pensé que estaba con el pañal sucio y podría estar incómodo y también para darle de lactar..." (MUI).

“...Yo ingresé al lactario por primera vez cuando mi hijito tenía 3 meses, ahora ya tiene 1 año, ingresé por la necesidad de cambiarle su pañalito y para de darle de lactar, porque estaba fastidioso..." (MU3).

“...Ingresé al lactario para dar de lactar y cambiarle el pañalito a mi bebe, pues sabemos que ellos después de alimentarse, hacen sus necesidades, yo aproveché el estar aquí para poder hacerlo..." (MU15).

Las unidades de significado, muestran claramente que las madres usuarias del lactario, hicieron uso adecuado vs un uso inadecuado del mismo. Al respecto, cabe mencionar que la necesidad de alimentar al bebé, va muy ligada con la necesidad de eliminación, puesto que existe un reflejo gastrocólico que se activa inmediatamente que el niño inicia la lactancia materna, cuyo efecto es que este realice la deposición después de mamar, esto conlleva a la necesidad imperiosa de cambiar el pañal, tan pronto la madre se dé cuenta, tal como lo haría en su propio hogar20; esto explica por qué las madres, realizan después del amamantamiento, el cambio de pañal, a pesar de que existe servicios higiénicos con cambiadores para bebés al costado del lactario, lo cual sería lo ideal, para no contaminar el ambiente.

Luego de haber descrito y analizado el uso que le vienen dando las madres al lactario, se encontró una serie de factores que limitan su uso, los mismos que se discuten en la siguiente categoría.

II. Limitantes para el uso del lactario: infraestructura inadecuada y desconocimiento de la madre usuaria:

Las limitantes, hacen referencia al conjunto de factores o condiciones, que se oponen al desarrollo de un proceso u acción determinada ${ }^{21}$. En primera instancia presentaremos los discursos que hacen referencia a la "infraestructura 
inadecuada", como limitante para el uso del lactario.

Cuando decimos "infraestructura inadecuada", nos referimos al incumplimiento de las condiciones estipuladas en la normativa referente a la implementación de lactarios en las instituciones del sector público y privado, a fin de promover la lactancia materna en los centros de trabajo, en el marco de las políticas de conciliación entre la vida familiar y las actividades laborales 4 .

El lactario del Centro Comercial Real PlazaChiclayo, es un espacio que no cuenta con las condiciones idóneas en infraestructura, establecidas en la normativa para su implementación y funcionamiento; las mismas, que no solo fueron evidenciadas por las investigadoras en las diferentes visitas realizadas al escenario para captar a los sujetos, si no también, fueron mencionadas por las madres usuarias, en sus discursos:

“... simplemente cuenta muebles y un cambiador para bebé, no es un lugar higiénico, por lo que se encuentra a lado de los servicios higiénicos, por donde pasa el personal de limpieza, no es un lugar acondicionado y amoblado para las madres que van a dar de lactar" ... no cuenta con cortinas. Casi siempre se observa al personal de limpieza en el lugar guardando productos (detergente y lejía) ..." (MU2).

“...solo tiene unos sillones, un tacho de basura y le falta muchas cosas, como, un friobar para poder sacarnos la leche, poder refrigerarla y luego el microondas para poder calentar. También necesita papel toalla, o para secarnos las manos, y bueno le falta muchas cosas más. Desinfectantes para las manos, porque uno cuando cambia al bebé del pañal uno tiene que desinfectarse las manos antes y después y para darle de lactar sin riesgo a infecciones..." (MU5).

"...Considero, conforme lo que he visto que es este ambiente que es demasiado cerrado, le falta ventilación, falta iluminación, y es un ambiente muy pequeño, solamente se podría usar un momento, ya que, si queremos usarlo por tiempo prolongado con nuestro bebé, es poco cómodo..." (MU10).

Según los relatos, podemos apreciar que en base a la experiencia que tuvieron las madres usuarias del lactario, ellas refieren que este, no cuenta con las condiciones necesarias de: higiene, confort y privacidad, para llevar a cabo el amamantamiento de sus bebés, e incluso otras prácticas de cuidado a las que le vienen dando el uso.

Cabe mencionar que, según la normativa ${ }^{4}$, el área mínima con la debe contar con un lactario, es de 10 metros cuadrados; respecto a la accesibilidad se mantiene el criterio de ubicación en el primer piso, o segundo, salvo que se cuente con ascensor; asimismo, se incluye la necesidad de considerar las adecuaciones que faciliten su uso en los casos de usuarias con discapacidad. Por otro lado, en relación a la descripción del equipamiento idóneo con el que debe contar, se menciona a la refrigeradora, además debe incluir también la alternativa del friobar, debido a que permite conservar la leche materna de las usuarias durante el horario de trabajo, constituyéndose como un elemento esencial para el uso exclusivo del lactario.

Además, se hace mención al lavabo, un dispensador de agua potable y demás utensilios de aseo que permitan el lavado de manos, a fin de garantizar la higiene durante el proceso de extracción de la leche materna. Cabe señalar, que también es de gran importancia hacer hincapié en la privacidad y comodidad del lactario. Incluso, existe la idea de que en los lactarios debería haber un teléfono o computadora, para que las 
empleadas puedan seguir trabajando en caso de querer hacerlo ${ }^{4}$.

Como podemos evidenciar, existe una gran diferencia entre la infraestructura del lactario y lo estipulado en la normativa vigente, verificada no solo por las investigadoras, si no también referida por los sujetos que participaron en la investigación, lo que hace deducir que este lactario, no presenta las condiciones necesarias en infraestructura, equipamiento y en privacidad, para el fin que está dirigido, a ello se agrega el desconocimiento de las madres sobre el correcto uso del lactario, condición que se agrava por la falta de difusión y educación al respecto.

Otra limitante para el uso del lactario, identificada por un grupo de madres usuarias, es el "desconocer la utilidad del lactario", condición que las conlleva a darle un mal uso, impulsadas por las necesidades emergentes de sus bebés y por imitación de lo que otros hacen, tal como se muestra en los siguientes discursos:

"...no se mucho de los espacios del lactario, pero al menos esto nos proporciona una pequeña intimidad, un pequeño espacio para poder dar de lactar sin que la gente nos esté mirando, poder aparte descansar o poder cambiar de repente un pañalito..." (MU4).

“.. me supongo que es para las madres que tienen sus bebés para poder cambiarles de pañal o darles de lactar..." (MU5).

“...bueno creo este lugar está hecho para las madres y sus bebes, funciona como un lugar en el que puedes aprovechar para hacer innumerables cosas, hasta para descansar..." (MU13).

Diversos estudios han demostrado a lo largo del tiempo, que el desconocimiento es un factor de riesgo para muchos comportamientos no saludables, que conllevan a grandes riesgos contra la salud ${ }^{22}$. Pues en este caso en específico, el hecho de que no se difunda la normativa vigente relacionada con la implementación y el uso del lactario en los diferentes medios de comunicación, ni en las instituciones de salud; además de la falta de monitoreo y supervisión de los pocos lactarios implementados a nivel nacional y local, explicaría el desconocimiento del uso adecuado de este servicio, situación que podría mejorarse, si existiera mayor difusión de todo lo relacionado con la práctica de la lactancia materna y la normativa vigente en cuanto a la implementación del lactario.

\section{Conclusiones}

Luego de haber realizado el análisis de los datos recolectados, las investigadoras, concluyen que el uso que le dan las madres usuarias al lactario público del Real Plaza Chiclayo, es adecuado e inadecuado, toda vez que existe un grupo minoritario de madres que le dan el uso correcto, acorde al propósito del lactario; en tanto que la mayoría le da un uso incorrecto, existiendo también un grupo de madres que le dan ambos usos; dejándose llevar por las necesidades emergentes de sus niños o por imitación de lo que los demás hacen en el ambiente, esto evidencia la necesidad urgente de difundir la normativa vigente sobre la implementación y funcionamiento de este servicio en la población, en particular con el público objetivo, las madres lactantes.

Por otro lado, existen condiciones que influyen en las madres usuarias, para no hacer uso adecuado del lactario y por ende no llevar a cabo la práctica correcta del amamantamiento en este espacio, estas son: la infraestructura y el desconocimiento, la mayoría de ellas refieren, que el lactario del Centro Comercial Real Plaza, posee una infraestructura inadecuada para satisfacer las necesidades de sus bebés. Por el contrario, una minoría refirió en sus discursos, conocer la finalidad del lactario, y más de la mitad 
evidenciaron desconocimiento acerca de la implementación y funcionamiento de este, como ambiente exclusivo de promoción de la lactancia materna. Todo ello, se ocasiona por el hecho de no contar con la información apropiada relacionada al uso correcto, y que a su vez no exista un monitoreo o supervisión de dicha área por parte de instituciones encargadas de la promoción de la lactancia materna.

\section{Bibliografía}

1. Coronado M, Sánchez O, Rodríguez A, Gorrita R. Causas de abandono de la lactancia materna exclusiva antes de los seis meses en san José de las lajas [Tesis doctoral en internet]. Filial ciencias médicas de la habana: Hospital general docente Leopoldito Martínez san Jose de las Lajas;2011.[consultada 18 de noviembre 2019].73 p. Disponible en: http://files.sld.cu/enfermeriapediatria/files/2011/03/lactanciamaterna.pdf

2. Fondo de las Naciones Unidas para Ia Infancia, (UNICEF), Lactancia Materna, citado el 09 de abril de 2012. Disponible en: http:/ / www.unicef.cl/lactancia/

3. Cerda L. La madre que amamanta y el entorno laboral. Matronas profesión [Internet]. Sept 2008[consultado 26 agos 2017];9(1):21-26. Disponible en:

http://www.federacionmatronas.org/resourceserver/192/d112d6ad $-54 \mathrm{ec}-438 \mathrm{~b}-9358-$ $4483 \mathrm{fge} 98868 / 82 \mathrm{~b} / \mathrm{rglan}$

4. Ministerio de la mujer y la población vulnerable MINP. Decreto Supremo que desarrolla la Ley No 29896 - Ley que establece la implementación de lactarios en las instituciones del sector público y del sector privado promoviendo la lactancia materna [Internet]. Disponible en: https://www.mimp.gob.pe/webs/mimp/lacta riosinstitucionales/pdf/rm_1282017mimp.pd f

5. Comisión Multisectorial de Lactarios. Informe anual de lactarios 2015 situación de los lactarios institucionales [internet]. Perú. Mar;2016[consultado $28 \quad$ Ago 2017].Disponible en: https://www.mimp.gob.pe/webs/mimp/lacta rios-institucionales/pdf/informe-anuallactario-2015.pdf

6. Ministerio de Salud (MINSA). "Perú es líder en lactancia materna en América Latina". Sala de prensa [Internet], Lima / Perú ;2015[consultado 28 Ago 2017]. Disponible en: http:/ / www.minsa.gob.pe $/$ ?op $=51$ \&nota $=16$ 807

7. Tojar J. Investigación cualitativa: Comprender y actuar. Madrid: La Muralla; 2006. 392p.

8. Flick $U$. La gestión de la calidad en investigación cualitativa. Madrid: Morata; 2014. 190p.

9. Lüdke M, Marli E. Pesquisa em Educação: Abordagens Qualitativas. E.P.U. Editora Pedagógica e Universitaria LTDA. André-São Paulo; 1986. Disponible en: file://C:/Users/Claudia/Downloads/312211555-1-PB\%20(1).pdf

10. Hernández R, Fernández C, Baptista L. Metodología de la Investigación. 6ta edición. México: Mc. Graw Hillinteramericana; 2014. 434p.

11. Denzin N. El campo de la Investigación cualitativa; $3^{\mathrm{a}}$ ed. Colombia; 2012. 346p.

12. Burns N, Kerouac S. Investigación en Enfermería. $3^{a}$ ed. España: ElSevier S A; 2009. 869p.

13. Sgreccia E. "Manual de bioética I. Fundamentos y ética biomédica". Principia. España: editorial BAC; 2014.992 p.

14. Noreña A, Moreno $\mathrm{N}$, Rojas J, Malpica D. "Aplicabilidad de los criterios de rigor y éticos en la investigación cualitativa". Colombia. 
[Internet]. Diciembre 2012.Vol 12. N³.

[consultado 26 de octubre 2017]. Disponible

en: [Internet]. Diciembre 2012.Vol 12. №3.

[consultado 26 de octubre 2017]. Disponible

en:

http://jbposgrado.org/icuali/Criterios\%20de \%20rigor\%20en\%20la\%20Inv\%20cualitativa.pd $\mathrm{f}$

15. Pérez J, Gardey A. definición de uso. [Internet] Publicado: 2010. Actualizado: 2012. [citado 17 Jun de 2018]. Disponible en: https://definicion.de/uso/

16. Real Academia de la Lengua Española. [Internet]. Actualizado 2017. [citado 17Jun de 2018]. Disponible en: http:/ / dle.rae.es/?id=0i9eAFV

17. Rodríguez A. Protocolo de normas y procedimientos de un lactario institucional. [Tesis de licenciatura]. [Bogotá]: Pontificia Universidad Javeriana; 2010. [Consultada 12 Jun 2018].23p. Disponible en: https://repository.javeriana.edu.co/bitstream /handle/10554/8618/tesis573.pdf?sequence $=1$

18. Organización Mundial de la Salud (OMS). Lactancia materna. Página Oficial de la Organización Mundial de la Salud [Internet]. Washington (DC): Oficina Regional para las Américas de la Organización Mundial de la Salud; 2007 [actualizado 1 agosto 2017; consultado 22 noviembre 2019]. Disponible en:

https://www.who.int/mediacentre/events/20 17/world-breastfeeding-week/es/

19. Núñez CH, Franco J, Labrador M. Impacto del lactario domiciliario en madres trabajadoras Hospital Central de San Cristóbal 2015. Venezuela. [Internet]. 2017. Vol. 80 (1): 10-14. [Consultado 11 Jun 2018]. Disponible en: http://www.redalyc.org/html/3679/3679518 39004/

20. Presilla R. Gastroenteritis. Updated: Apr 25, 2008. Disponible en: http://emedicine. medscape.com/article/ 964131 -overview

21. Rodríguez G. DIRAE. definición de limitante. [Internet] Publicado: 2017. Actualizado: 2017. [citado 22 Jun de 2018]. Disponible en: https://dirae.es/palabras/limitante

22. Valenzuela. S, Vásquez. E \& Gálvez P." Factores que influyen en la disminución de lactancia materna exclusiva hasta los 6 meses de vida". Rev int salud materno fetal. Chile.2016: 1 (7).[acceso 22jun de 2018].Disponible en: http://repositorio.uchile.cl/bitstream/handle /2250/143158/Factores-queinfluyen. pdf?sequence $=1$ \&isAllowed $=y$ 